\title{
THE GROUP OF A TACTICAL CONFIGURATION.
}

BY PROFESSOR L. E. DICKSON.

(Read before the American Mathematical Society, December 29, 1904.)

1. A tactical configuration, connected with the abelian group $G_{p^{m}}$ of type $(1,1, \cdots, 1)_{m}$ and serving to define the general linear homogeneous group $H$ modulo $p$ on $m$ variables, has been given by Professor Moore.* As an obvious generalization, consider the configurations defining the various subgroups of $H$. The example that I give had its origin in the following problem: $\uparrow$ Required the number $N_{n}$ of all possible ways of separating the $2^{2 n}-1$ operators other than identity of $G_{2^{2 n}}$ into $2^{n}+1$ sets each of $2^{n}-1$ operators, such that the operators of any set together with identity form a subgroup $G_{2^{n}}$ and such that no two sets have a common operator. Here $G_{2^{2 n}}$ is assumed to be an abelian group of type $(1,1, \ldots, 1)_{2 n}$; for example, the group of all linear transformations on $2 n$ variables which multiply each variable by \pm 1 .

2. That such a separation is always possible is easily shown. The group of automorphisms of $G_{2^{2 n}}$ may be taken concretely as the group of all linear homogeneous transformations modulo 2 on $2 n$ variables. The latter contains $\$$ a transformation $S$ whose characteristic equation of degree $2 n$ is irreducible modulo 2 , so that $S$ is of period $2^{2 n}-1$. In particular, an operator $\Sigma$ of period $2^{n}-1$ occurs. Let $I, a_{1}, b_{1}, a_{1} b_{1}, \ldots$ be the operators of a subgroup $G_{2^{n}}$ of $G_{2^{2 n}}$. A table of the operators of the latter may be formed with those of $G_{2^{n}}$ in the first row and with the operators $I, a_{2}, b_{2}, \ldots$ of a second $G_{2^{n}}^{\prime}$ as multipliers. We may choose $\Sigma=\Sigma_{1} \Sigma_{2}$, where $\Sigma_{i}$ permutes cyclically the $2^{n}-1$ elements $a_{i}, b_{i}, a_{i} b_{i}, \cdots$, written in a suitable order. As the first set $S_{1}$ we take $a_{1}, b_{1}, a_{1} b_{1}, \cdots$; as the second set $S_{2}$ we take $a_{2}, b_{2}, \ldots$ To form the third set $S_{3}$, take any element, as $a_{1} a_{2}$, in neither $S_{1}$ nor $S_{2}$, and apply to it the powers of $\Sigma$; there result $a_{1} a_{2}, b_{1} b_{2}, \ldots$. To form the $i^{\text {th }}$ set take any element not in $S_{1}, S_{2}, \cdots, S_{i-1}$ and apply to it the powers of $\Sigma$. In this way we obtain $2^{n}+1$ sets with the desired properties.

* Bulletin, vol. 2 (1895), pp. 33-43.

+ For $n=2$, see Burnside, Theory of Groups, p. 60, ex. 2 ; errata, p. xvi.

$\ddagger$ Linear Groups, p. 236. 
3. For $n=2$, we take $\Sigma=(a, b, a b)(A, B, A B)$ and obtain

$$
\begin{gathered}
S_{1}=\{a, b, a b\}, S_{2}=\{A, B, A B\}, S_{3}=\{a A, b B, a b A B\}, \\
S_{4}=\{b A, a b B, a A B\}, S_{5}=\{a b A, a B, b A B\} .
\end{gathered}
$$

A suitably chosen substitution of period 5 on the 15 letters (\$2) will permute the $S_{i}$ in a cycle. The following substitutions leaving $a$ and $b$ unaltered: $(A, a A)(B, b B)$, ${ }^{*}$ ( $A, B, A B)$ induce on the $S_{i}$ the substitutions $\left(S_{2} S_{3}\right)\left(S_{4} S_{5}\right)$ and $\left(S_{3} S_{5} S_{4}\right)$, respectively. Finally, $(a, b)(A, B)$ induces $\left(S_{4} S_{5}\right)$. Hence the $S_{i}$ may be permuted in all 120 ways. Next, in view of their origin, each set $S_{i}$ is unaltered by $\Sigma$ and its powers, but by no further substitution. Indeed, if each $S_{i}$ is unaltered by $T$, we have $T=T_{1} T_{2}$, where $T_{1}$ affects the $a, b, a b$ in the same way that $T_{2}$ affects $A, B, A B$. But if the $T_{i}$ are transpositions, $S_{4}$ and $S_{5}$ are permuted.

The group of the configuration $S_{1}, \cdots, S_{5}$ is an imprimitive $G_{360}^{15}$ which gives rise to all 120 permutations of the $S_{i}$.

4. For $n=3$, we take as $\Sigma$

$$
(a, a b c, c, b c, a b, b, a c)(A, A B C, C, B C, A B, B, A C) \text {, }
$$

the first seven elements forming $S_{1}$, the second seven forming $S_{2}$. The remaining sets are formed as in $\S 2$ :

$$
\begin{aligned}
& S_{3}=\{a A, b B, a b A B, c C, a c A C, b c B C, a b c A B C\}, \\
& S_{4}=\{c A, a B, a c A B, a b C, a b c A C, b B C, b c A B C\}, \\
& S_{5}=\{a b A, c B, a b c A B, a c C, b c A C, a B C, b A B C\}, \\
& S_{6}=\{a c A, a b B, b c A B, a b c C, b A C, c B C, a A B C\}, \\
& S_{7}=\{a b c A, a c B, b A B, b c C, a A C, a b B C, c A B C\}, \\
& S_{8}=\{b A, b c B, c A B, a C, a b A C, a b c B C, a c A B C\}, \\
& S_{9}=\{b c A, a b c B, a A B, b C, c A C, a c B C, a b A B C\} .
\end{aligned}
$$

Thus $\Sigma$ leaves each $S_{i}$ unaltered. Any substitution $T$ on the 63 letters which leaves unaltered each $S_{i}$ is a power of $\Sigma$. In fact, $T$ must affect the large letters in the same way that it does the small, in view of $S_{3}$. Now $T \Sigma^{s}$, where $s$ is suitably chosen,

\footnotetext{
* The further cycles $(A B, a b A B)(b A, a b A)(a B, a b B)(a A B, b A B)$ are suppressed. The shorter notation suffices as it gives the new generators. The same remark applies throughout.
} 
will leave $a B$, and hence $a, B, A, b$, unaltered. But $c A$ occurs in $S_{4}$, so that $c$, and hence $C$, are unaltered. Hence $T \Sigma^{s}$ is the identity.

Next $S_{1}$ may be thrown into any $S_{i}$. Further,

$$
(A, a A)(B, b B)(c, c C), \quad(A, A B C, C, B C, A B, B, A C),
$$

each leaving $a, b, c$ unaltered, induce on the $S_{i}$ the substitutions

$$
\left(S_{2} S_{3}\right)\left(S_{4} S_{6}\right)\left(S_{7} S_{9}\right)\left(S_{5} S_{8}\right), \quad\left(S_{3} S_{6} S_{8} S_{5} S_{9} S_{4} S_{7}\right),
$$

respectively. Hence the group induced on the $S_{i}$ is triply transitive. The following substitution, leaving $S_{1}, S_{2}, S_{3}$, a, and $A$ unaltered,

$$
(b, b c, a c)(c, a b, a b c)(B, B C, A C)(C, A B, A B C)
$$

induces the substitution $\left(S_{4} S_{5} S_{7}\right)\left(S_{6} S_{8} S_{9}\right)$. But there is no substitution $T$ corresponding to one leaving fixed $S_{1}, S_{2}, S_{3}$, and replacing $S_{4}$ by $S_{6}, S_{8}$, or $S_{9}^{\gamma}$. Employing $T \Sigma^{r}$ instead of $T$, where $r$ is suitably chosen, we may suppose that $a$ and $A$ are also fixed. Let then $T$ replace $S_{4}$ by $S_{6}$. Hence $T$ replaces $c$ by $a c$, and $B$ by $A B C$, in view of the coefficients of $A$ and $a$ in $S_{4}$ and $S_{6}$. But $S$ must affect the large letters in the same way that it affects the small, in view of $S_{3}$. Hence $T$ replaces $C$ by $A C$, and $b$ by $a b c$. Hence $T$ replaces $a b C$ of $S_{4}$ by $b c A C$, not in $S_{6}$. Similarly, $T$ cannot replace $S_{4}$ by $S_{8}$ or $S_{9}$. Finally, an induced substitution which leaves $S_{1}, S_{2}, S_{3}, S_{4}$ each fixed, leaves every $S_{i}$ fixed and is the identity.

The group of the configuration $S_{1}, \ldots, S_{9}$ is an imprimitive $G_{1512.7}^{63}$ which gives rise to a triply transitive $G_{1512}^{9}$ on the $S_{i}$.

5 . Since the $2 n$-ary linear homogeneous group $H$ modulo 2 has the order

$$
\left(2^{2 n}-1\right)\left(2^{2 n}-2\right)\left(2^{2 n}-2^{2}\right) \cdots\left(2^{2 n}-2^{2 n-1}\right),
$$

we conclude that $N_{2}=2^{3} .7, N_{3}=2^{12} \cdot 3.5 .31$. In fact,* every separation of the required kind is conjugate within $H$ with that obtained by $\Sigma$ in $\S \S 3-4$.

University of Chicago, October, 1904.

\footnotetext{
* A direct proof is given in the Amer. Math. Monthly, Nov., 1904.
} 